Poétique et politique dans le théâtre de Michel Vinaver

\author{
Gina Basta \\ Universite Ain Shams \\ Faculte Al Alsun
}

\title{
Poétique et politique dans le théâtre de Michel Vinaver
}

(Au théâtre) les peuples démocratiques ne se soucient guère de ce qui se passait à Rome et Athènes

; ils entendent qu'on leur parle d'eux-mêmes, et c'est le tableau du présent qui les intéresse. Tocqueville $^{1}$

Les rapports de la poétique et de la politique ont existé depuis que fut connue l'écriture. Sans être expressément engagé, tout écrivain, digne de ce titre, est mû par une problématique ou une cause qui l'incite à réagir soit par opposition, soit par adhésion. Parmi les divers genres littéraires, le théâtre qui est, de par sa nature même lié à la vie sociale, semble un cadre privilégié pour y exposer,

\footnotetext{
${ }^{1}$ TOCQUEVILLE A., « De la démocratie en Amérique $2 », 1840$, in Les Classiques de sciences sociales, éd. PhiloSophie, Chapitre 2, mai 2008.
} 
jouer ou débattre les enjeux politiques. C'est un merveilleux miroir de la société. Pour le définir, il importe d'interpréter la dimension politique ancrée dans le rôle même du théâtre : il est fournisseur et interprète d'informations. Il présente obligatoirement un parti pris et offre ainsi une interprétation implicite de cette position partiale : "Plutôt que de se demander comment le théâtre peut être politique, ne vaudrait-il pas mieux réfléchir au fait qu'il l'est, en quelque sorte, ontologiquement ${ }^{2}{ }^{2}$

La notion de «théâtre politique » présente divers aspects, depuis le contenu qui «sert tout chauds» des incidents, tirant profit de la vivacité propre à l'immédiat, jusqu'à la forme qui fait usage de tous les outils disponibles : aussi bien la dramaturgie que les arts de la scène afin d'impressionner, de produire, poétiquement, un effet qui traduirait la position de l'auteur à l'égard de telle ou telle question. Ayant déterminé le concept de ce théâtre, il reste à clarifier ce qu'on entend par le qualificatif «politique » avant d'entreprendre l'étude du théâtre politique de Michel Vinaver.

En effet, «la politique » marque tout ce qui se rapporte aux œuvres des gouvernements, des partis, des organismes...etc. ${ }^{3}$, enfin tout ce qui forme la patrie. Or, cette conception ne prend pas en compte «le politique » comme engagement ou prise de position quotidienne et intérêt personnel. Elle n'implique pas non plus le côté économique ni l'interaction populaire ou les relations conflictuelles des groupes humains partageant une même société. Par conséquent, c'est cette notion du politique qui se reflète dans l'engagement littéraire, même si toute littérature n'est pas forcément une fidèle reproduction d'un incident politique bien défini. De là, la poétique se trouve en rapport direct, non seulement avec « la politique », mais surtout avec « le politique ».

Cette vision du politique a pris un essor considérable dans la deuxième moitié du XXème siècle. Elle propose différents critères pour introduire «le politique » dans une ambiance de théâtre qui n'est guère délibérément politique. Racisme, préférences sexuelles ou orientations de la consommation, ont été reconnus autour du milieu du siècle précédent comme l'impact dynamique du politique. ${ }^{4}$ Ainsi, toute dramaturgie devient politique puisque toute pièce transmet au moins une information partielle et en offre une interprétation. Le corollaire est également vrai: une pièce qui ne critique pas la conjoncture sociopolitique contribue à son maintien et lui sert de support. $^{5}$ Cette étude se propose donc d'examiner la dimension politique de l'un des

\footnotetext{
${ }^{2}$ DORT B., (1986), Théâtres, Paris, Seuil, p.233.

${ }^{3}$ cf. KIRBY M. (1975), “On Political Theatre”, in The Drama Review, n 19, June, p.129.

${ }^{4}$ cf. MARKOVIC M., (1974), The Contemporary Marx: Essays on Humanist Communism,

Bristol, New Spokesman Press, p.173.

${ }^{5}$ RABEY D., (1986), British and Irish Political Drama in the Twentieth Century, London,

MacMillan, p.1.
} 
dramaturges français contemporains les plus remarquables, Michel Vinaver, à travers deux pièces:

Les Coréens (mise en scène en1955) est sa première pièce où il débutait sa carrière dramaturgique. La toile de fond de cette pièce est tissée par un événement politique et militaire : les péripéties de la guerre de Corée (19501953), où le public s'étonne de voir Belair, un caporal français, changer de camp et de convictions. Il est membre d'un bataillon de six soldats français, ayant pour mission le bombardement d'un village coréen ; mais il est blessé à mort. Malgré les décombres et les dégâts, le village se rétablit, tentant de reprendre une vie quotidienne normale. Une fillette de huit ans du village, à la recherche du cadavre de son frère fusillé par les soldats ennemis, découvre le caporal blessé, le ramène au village, où il est soigné, hébergé. Simultanément, les soldats français du bataillon, perdus dans les forêts tropicales, échangent leurs uniformes avec ceux des soldats morts du camp ennemi. D'ailleurs, la pièce commence par la réplique d'un paysan coréen qui dit à un soldat : Qu'estu venu faire dans ma maison? En effet, les soldats français se posent la même question, que sont-ils venus faire dans ce pays dont ils ne savaient même pas l'existence?

La seconde pièce, 11 Septembre 2001, est, par contre, une œuvre de fin de carrière, pièce relativement récente, écrite dans l'immédiat d'un événement également politique : l'attentat terroriste de deux avions américains détournés et dirigés pour s'écraser dans les deux tours newyorkaises «Twin Towers », entraînant leur chute, au centre de New York. Ils visaient ainsi le cœur du système économique des États-Unis. Cet attentat fait basculer la politique mondiale du début du troisième millénaire. Vinaver choisit pour titre la date de cet incident afin qu'elle demeure gravée dans les consciences. Il dit avoir voulu "réfléchir l'événement plutôt qu'y réfléchir ${ }^{6}{ }$.

Ayant chacune un caractère spécifique, les deux pièces témoignent de la volonté de Vinaver de faire face aux événements actuels au fur et à mesure qu'ils se produisent. Deux modèles qui s'inscrivent directement dans la tradition du théâtre de l'immédiat, soucieux de mettre en scène la conjoncture : un théâtre qui traite aussi bien des incidents terroristes que des problèmes politiques et sociaux, tout en désignant leurs substrats. Ces deux pièces, sans être explicitement subversives, s'attaquent aux conditions politiques et sociales qui engendrent cet état de choses.

Le parcours de Michel Vinaver se compose d'une vingtaine de pièces au cadre apparemment conventionnel du drame bourgeois, aux tableaux disloqués

${ }^{6}$ DIAZ S. , « 'Réfléchir l'événement plutôt qu'y réfléchir' : l'imitation du 11 septembre 2001 dans la pièce de Michel Vinaver», Agôn [En ligne], Dossiers, (2011) HS n ${ }^{\circ} 1$ : Mettre en scène l'événement, 11 Septembre 2001 de Michel Vinaver, mis à jour le : 31/10/2011, consulté 18/11/2017.

URL : http://agon.ens-lyon.fr/index.php?id=1803. 
et interrompus, portant en filigrane les pesanteurs socio-économiques sousjacentes dans la société occidentale. Il entend signaler leurs retombées sur la vie du citoyen ordinaire ; comme pour l'inviter à les reconsidérer en les immobilisant devant ses yeux. Puis, l'auteur se détourne de l'histoire, laissant s'évanouir les thèmes qui nourrissent le théâtre bourgeois, ne permettant pas à l'intrigue d'arriver à une conclusion satisfaisante. Le fond politique occupe le premier plan, laissant à l'auditoire l'occasion de tisser les rapports entre les personnages et le développement du système social et économique.

Un des éléments traditionnels du théâtre bourgeois réside dans le divertissement de l'auditoire sans chercher à saper 1'ordre des choses ; Vinaver en profite pour induire son spectateur passif dans un faux sentiment de sécurité, pour le secouer ensuite par la prise de conscience de la réalité.

\section{Caractéristiques du théâtre politique:}

On pense d'une manière générale que le théâtre politique est un théâtre subversif qui pourrait perturber le conventionnalisme et prêter à la révolution. Il n'en est rien : en soulevant certaines causes, ce théâtre ne fait que dévoiler des problèmes sous-jacents et poser des questions ; il ne présente pas de solutions aux problèmes soulevés, n'appuie pas nécessairement des alternatives particulières aux dépens d'autres : susciter un changement d'opinion n'est pas l'unique tâche du théâtre politique. Mais il pourrait fournir une force motrice, un appui émotionnel et intellectuel pour le changement politique, en soutenant une certaine prise de conscience plutôt que d'en causer ou d'en produire une. D’après Vinaver, la tâche du théâtre politique se résume en ces mots :

"préparer le champ à l'invention de nouveaux usages; et d'en trouver le moyen, qui est de susciter une émotion "délivrante» chez le spectateur, une émotion qui dénoue ses réflexes défensifs, le délie de ses habitudes et de ses fidélités. ${ }^{7}{ }^{\prime \prime}$

Vinaver affirme l'importance de cette «émotion délivrante » qui met le spectateur face à sa propre réalité : «ce que dans sa propre vie il emploie ses efforts à éviter. ${ }^{8}$ »

D’autres types de théâtre interrogent la notion de « patrie ». Le patriotisme est un sentiment d'appartenance à une nation, concrétisé dans les institutions et les croyances partagées par la communauté politique. Une nation montre souvent son dévouement patriotique par l'hommage rendu aux monuments de ceux qui sont morts pour le pays. Or la première création dramatique de Vinaver tourne en dérision cette pratique et en fait un jeu grotesque ; ce qui remet en question la crédibilité de l'institution de la guerre et des idéaux

\footnotetext{
${ }^{7}$ VINAVER M., (1998), Ecrits sur le théâtre I, (1998). Paris, L’Arche, p.36.

${ }^{8}$ Ibidem
} 
constituant la patrie. Dans Les Coréens, les soldats français simulent un « jeu de monument » qui bouleverse la vision officielle de cette notion. Lhomme et Lhorizon (deux soldats français) capturent un jeune rebelle du Nord de Corée, qui avait l'habitude de poser des mines au bord des routes. Ils l'attirent au jeu, le placent au milieu, jettent un drap sur lui pour en faire un monument. Beaugeron inaugure le jeu en ces termes:

«Je vous invite à vous incliner bien, bas, très bas, devant la Mémoire de ceux qui sont morts pour beaucoup de choses à la fois, tellement de choses que tout ça, ça se mélange un peu, forcément. D'abord pour leurs aïeux. Et puis pour la gloire. Et puis, pour la liberté chérie. Et puis pour les arbres de leur pays. ${ }^{9} "$

Bientôt, cet éloge conçu en vue d'honorer le souvenir des camarades sacrifiés pour la gloire de la patrie dégénère en une scène ironique tournée en dérision par l'interrogation absurde des soldats qui se demandent ce qu'ils sont venus faire en Corée:

«Bonassier: On leur avait promis une prime.

Lhorizon: Qui sont morts parce qu'ils savaient pas

Exaxerguès: Qu'est-ce qu'ils savaient pas Lhomme : Il y en a qui sont morts parce qu'ils en avaient envie. Parce que la vie est une vraie chérie.

Beaugeron: Incline-toi, Exaxerguès! Qui sont morts, ça c'est sûr. Qui sont morts.

Exaxerguès : T’as pas dit où.

Beaugeron: Qui sont morts en Corée. Qu'est-ce qu'ils allaient faire en Corée? Tous:(à la fois et dans le fou rire). Qu'ça leur fichait... morts pour... Qu'ça leur chantait... Morts parce que... Oh pour!... Oh parce que!... Morts où! ... Oh hou !... ${ }^{10} \gg$

La présence des soldats français en Corée est justifiée comme une « défense du monde libre», une extension de la défense du pays mais les principes du jeu sont intervertis. Le monument dévoilé (qui est joué par le coréen capturé) accentue l'ambiguïté des relations entre les deux nations. D'une part, ce monument ne peut représenter le sacrifice des soldats français ; d'autre part, cela pourrait servir plutôt de cérémonie pour rendre hommage aux soldats coréens exterminés par les Français dont la présence n'est guère justifiable. De telles incongruités présentent la «patrie» comme une notion fragmentée. Dans Les Coréens, l'amitié entre un soldat français et une paysanne coréenne pro-communiste et les échecs successifs des troupes françaises pendant la guerre dans la jungle coréenne, remettent en question l'image de l'armée en tant que protectrice de la patrie, émancipatrice du peuple, incarnation de la volonté

\footnotetext{
${ }^{9}$ ID, (1986), Les Coréens, in Théâtre Complet I, Actes Sud et L'Aire, p.57.

${ }^{10}$ Ibidem
} 
de la nation. Dans cette pièce, Michel Vinaver crée une histoire à multiples facettes qui défie la vision cohérente de la patrie et sape certains concepts traditionnels de l'unité et de l'objectif national. En effet, Roland Barthes règle cette controverse en répondant à la question cherchant à élucider le caractère politique du théâtre de Vinaver :

«Non, si la politique, c'est le discours, la profession de foi, la thèse [...] : les personnages de Vinaver ne parlent jamais sur la politique. Oui, pleinement oui, si la politique consiste à retrouver les rapports réels des hommes débarrassés de toute « décoration » psychologique [...]. ${ }^{11}$ »

Or, pour jouer efficacement son rôle, la dramaturgie politique est consciente du besoin d'engager l'audience non seulement à travers le contenu de la pièce, mais aussi

à travers

la

forme.

Le XXème siècle a vu surgir une évolution sans précédent au théâtre, accompagnée souvent de développement de la forme, qui reflète les agitations politiques. A mesure que ces dernières changent de moyens et de modes de protestation, le théâtre aussi modifie ses moyens et modes d'expression.

«En France, de l'après 1968, par exemple, on a vu se produire une emphase parmi les activistes politiques sur les modes de produire des changements au niveau externe des partis politiques traditionnels. Mouvements pacifistes, étudiants, antiracistes, féministes s'étaient organisés superficiellement dans le but de fuir jusqu'à un certain point les formes caractéristiques des unions et partis politiques. ${ }^{12} \gg$

Simultanément, dramaturges et troupes théâtrales ont tenté de transformer les rapports liant l'auditoire à la scène. Ils présentent des pièces en étroite collaboration avec la population locale, organisent des discussions avec l'auditoire, puis intègrent ses points de vue dans des performances ultérieures. Ces changements dans la forme théâtrale favorisent la prise de conscience des problèmes, en impliquant directement le public dans des activités créatives et critiques. Cela encourage les spectateurs à prendre une part plus active pour envisager la politique personnelle ou locale : «Le théâtre est ce qui se passe entre spectateur et acteur ${ }^{13}{ }^{\prime}$.

Dans sa critique des Coréens, Emile Copfermann souligne que l'élément subversif, bien que diffus, semble assez puissant dans le théâtre de Vinaver : "Ecrite dans une langue claire, directe, efficace, sans ces schématismes qui guettent l'œuvre de "circonstance » en évitant l'écueil du manichéisme, en cela comme en son découpage épique, la «comédie» de Michel Vinaver devrait

\footnotetext{
${ }^{11}$ BARTHES R., (1956) «Aujourd'hui ou les Coréens » (de M. Vinaver, mise en scène par R.

Planchon, au théâtre de la Comédie), in France-Observateur, $1^{\mathrm{er}}$ nov.

${ }^{12}$ ELSTOB K., (1992), The Plays of Michel Vinaver. Political Theatre in France, NYC, American University studies, Ed. Peter Lang, p.3.

${ }^{13}$ GROTOWSKI J. \& FLASZEN L., (1967), L'Institut de recherche sur le jeu de l'acteur, Wroclaw, éd. du Théâtre Laboratoire, p.31.
} 
connaître un accueil favorable du public. Mais faudrait-il trouver quelqu'un qui osât la montrer. ${ }^{14}$ "

En attirant l'attention sur les problèmes politiques, on constate que la forme est aussi importante que le contenu puisqu'elle " se situe entre le matériel et l'idéal ; le monde objectif des choses concrètes et le monde mental de la pensée et des idées. La forme confère une puissance empirique à l'état intellectuel, tout comme les mots et les actions. ${ }^{15}$ "

Les préparatifs de la représentation des Coréens impliquent aussi une note subversive: les soldats français sont perdus dans un environnement géographique particulier, pour lequel Roger Planchon, metteur en scène « $a$ fourni une image scénique de l'immédiateté de l'expérience en couvrant sa scène de sable et en la plantant dans une forêt de joncs et de buissons. ${ }^{16}{ }{ }$ Cette impression concrète de la forêt coréenne frustre les troupes françaises, qui ont appris à défendre la patrie dans un cadre plus conventionnel. Dans le feu d'une attaque, Exaxerguès, l'un des soldats du bataillon français, se demande si le peloton est derrière les lignes ennemies, puisqu'il ne peut voir aucune marque. Tout ce qu'il voit, ce sont des fleurs, pour lesquelles on a habituellement besoin de «tondeuses et non de fusils». En cherchant de nouvelles formes pour le théâtre, de nouveaux modes de spectacle on contribue à changer l'idée de l'auditoire à l'égard du monde de la politique. Pendant que le théâtre politique change, l'ordre établi qu'il entend troubler change aussi. Ainsi, le théâtre est lié au contexte historique. Voici comment le contexte historique de l'incident constitue un point à considérer. Une fois que le contexte historique est connu, la question ne concerne plus uniquement les messages de l'auteur dramatique mais toute la pratique théâtrale. Le théâtre n'est plus seulement l'ouvrage dramatique écrit, son contenu et sa forme, mais aussi la pièce telle qu'elle est présentée devant un certain public qui considère le travail en sa forme scénique:

"la question ne serait plus de savoir quelle peut être, dans des circonstances données, l'efficacité de telle ou telle cuvre dramatique, mais d'établir clairement la dimension politique qui est celle de tout grand thêâtre, quitte à évaluer, par la suite, comment auteurs et metteurs-en-scène aujourd'hui acceptent ou refusent une telle dimension. ${ }^{17}$ "

\section{Influences préalables :}

L'activité de Vinaver au théâtre pendant les premières années de sa carrière révèle les influences latentes dans ses approches dramaturgiques. Celles-ci sont incarnées dans un théâtre de lucidité subversive où s'entrelacent les péripéties

\footnotetext{
${ }^{14}$ COPFERMANN E., (1958), «Iphygénie Hôtel », in France-Observateur, 24 avril, p.10.

${ }^{15}$ ELSTOB K., (1992), op.cit. p.3.

${ }^{16}$ BRADBY D., (1984), Modern French Drama (1940-1980), Cambridge and London,

Cambridge University Press, p.108.

${ }^{17}$ DORT B., (1986), op.cit., p.233.
} 
de la vie quotidienne avec l'histoire politique contemporaine. Au cours de sa longue carrière qui a duré plus d'un demi -siècle, nous pouvons repérer certains aspects communs ou des oppositions avec ses prédécesseurs. Le lien entre économie et politique est un thème central dans l'ensemble de son œuvre dramatique. Vinaver a vu de ses propres yeux l'effet de l'économie sur les individus, pendant la période où il travaillait pour la société Gillette : "c'est de plus en plus par l'économique [...] que les gens tissent leur lien au monde. ${ }^{18}$ » Il semble préoccupé par l'impact du pouvoir libéral. Sa position de gauche évidente, bien que non définie, cherche à en saper le principe de fonctionnement «De gagner ma vie ainsi me permet de faire un théâtre sans souci [...] de rendement. Ce qui augmente ses chances d'être un théâtre d'innovation, donc de mise en cause du Système. Je n'essaie pas d'éluder la contradiction. Elle est le feu central. ${ }^{19}{ }^{»}$

Il dramatise thématiquement la condition économique à la manière du théâtre épique de Brecht qui prétend sauver son spectateur : "La douleur de cet être me bouleverse parce qu'il y aurait tout de même une issue pour lui. ${ }^{20}{ }{ }^{2}$ En effet, Vinaver admet que Brecht l'a influencé: «Brecht $m$ 'a fortement marqué2l . » Comme lui, il divertit ses spectateurs en les invitant à adopter une attitude critique, en présentant le familier sous un jour nouveau. Il essaie de les détourner de la position passive de « voyeurs ${ }^{22}$ », " de les faire déboucher d'une image inattendue d'eux-mêmes, et partant, d'agir sur l'histoire. ${ }^{23}$ »

D'autre part, la nature épisodique de ses pièces est aussi conforme à la technique brechtienne. Il montre le monde comme un processus continu de « devenir » plutôt que fixé dans des limites établies et immuables: "L'avenir du théâtre est lié au devenir du public, à la transformation radicale de la société. $^{24} \gg \mathrm{C}$ 'est ainsi que le théâtre, en tant qu'art évolutif, accomplit simultanément sa propre révolution. Pourtant, Vinaver s'oppose à Brecht, qui pense que la relation critique entre le public et le spectacle doit être réalisée par l'excès de théâtralité se dégageant du plaisir et d'un sentiment d'aliénation: "Le spectateur du théâtre épique dit : je n'aurais imaginé une chose pareille; on n'a pas le droit d'agir ainsi. Voilà qui est curieux, c'est à n'en pas croire ses yeux [...]: tout y est choquant, Je ris de celui qui pleure, je pleure de celui qui rit. $^{25}{ }$,

${ }^{18}$ VINAVER M., (1998), op.cit., p.286.

${ }^{19}$ Ibid, p.304.

${ }^{20}$ BRECHT B., (2000). «Théâtre récréatif ou théâtre didactique ? », in Ecrits sur le théâtre, Paris,

Galllimard, Bibliothèque de la Pléiade, p.214.

${ }^{21}$ VINAVER M., (1998), op.cit., p.306.

22 JEAN G., (1977), Le Théâtre, Paris, Seuil, p.163.

${ }^{23}$ VINAVER M., (1998), op.cit., p.105.

${ }^{24}$ JEAN G., (1977), op.cit., p.162.

${ }^{25}$ BRECHT B., (2000), op.cit., p.214. 
En revanche, il rejette les principes de ce théâtre épique, n'utilise pas d'effets d'aliénation ; ses pièces ne favorisent guère les fables et ne sont que très peu discursives.

Mais il adopte un autre point de départ qui : «consiste à prendre des éléments de réalité brute, plate, et à les dissocier les uns des autres en les recomposant par la méthode du montage, du collage, de l'assemblage, du lacérage. ${ }^{26} \gg$. C'est pourquoi sa conception de la réalité semble être assez marginale : «où il ne s'agit pas de reproduire la réalité, mais, par l'usage de signes et de symboles, de faire apparaître l'évidence (tragique, comique) de ce qui fonde la destinée de l'homme sur la terre. ${ }^{27}$

Barthes trouve que si le théâtre de Vinaver n'est pas à proprement dit réaliste, il est au moins «objectif» éloigné du psychologisme bourgeois et que «cette vue du réel est quelque chose de nouveau, je crois dans notre théâtre français. ${ }^{28}$ » Il cherche à perturber l'ordre des choses par «un travail de "frottement » des éléments les uns aux autres, de glissement, d'entrechocs ${ }^{29}$ », utilisant une structure épisodique qui empêche le consentement passif au récit linéaire facilement digéré et insiste sur la fonction exploratoire du théâtre, «le volontarisme dans l'art est mutilant. La réalité c'est l'homme qui la constitue en l'interrogeant. Notamment par l'activité artistique ${ }^{30}{ }$, dit-il.

Or, la conception dramatique brechtienne n'est pas la seule qui aurait laissé ses empreintes sur le théâtre politique de Vinaver, les techniques dramatiques de Beckett et d'Ionesco, qui ont radicalement défié les formes conventionnelles, l'ont également influencé. Ces dramaturges ont encouragé leur public à abandonner la position privilégiée de spectateur et à connaître les confusions et les ambiguïtés imposées par le pouvoir. En outre, Vinaver est amateur du théâtre grec et classique, et possède une longue expérience dans la traduction de ce théâtre ainsi que les adaptations modernes des mythes grecs. Cette tendance semble conservatrice et peut risquer de le mettre à l'index par rapport aux innovateurs de son époque; mais il n'en est rien car le dramaturge repousse le contrôle exercé par le théâtre conservateur sur son public: «les formes dramatiques servent à rassurer quand il s'agirait d'ébranler, à conserver quand il faudrait faire table rase. ${ }^{31}{ }$. Selon lui, l'aura qui cerne les ouvrages provenant de la Grèce ancienne donne un ton stable soit aux reproductions modernes des tragédies grecques, soit aux adaptations théâtrales des mythes

${ }^{26}$ VINAVER M., (1998),op.cit., p.313.

${ }^{27}$ Ibid, p.30.

${ }^{28}$ BARTHES., (1956). op.cit.

${ }^{29}$ ELSTOB K., (1992), op.cit., p.33.

30 VINAVER M., (1998), op.cit., p.306

31 Ibid, p.36. 
grecs. Vinaver regarde le théâtre et la mythologie grecs comme des sources de contradiction fertile :

"S'il est vrai que le mythe, vu de dehors, évolue incessamment et se transforme au fur et à mesure que se modifie la structure de la société, il n'en est pas moins vrai que saisi dans chacun de ses instants vécus il se présente sous un aspect immuable et définitif. ${ }^{32}{ }$ ”

Les ouvrages de l'ancien théâtre grec pourraient donner une appréciation sur le présent, comme dans le cas de La Guerre de Troie n'aura pas lieu (1935), où Giraudoux associe la situation politique à Troie, lorsque les Troyens sont en instance de guerre avec les Grecs, à celle contemporaine en Europe des années 1935, où l'Allemagne se préparait à la guerre contre la France.

Dans Antigone, Anouilh se concentre sur l'interrelation entre le pouvoir politique et la routine quotidienne. Dans l'adaptation d'Antigone par Vinaver, les acteurs portaient les costumes conçus pour Les Coréens (uniformes de l'armée française et costume paysan coréen). Les costumes évoquent le rôle de l'armée française en Algérie, en Indochine et en Corée. Bien distincte, la pièce témoigne du désir de Vinaver de gérer les événements actuels au fur et à mesure qu'ils se produisent. ${ }^{33}$

D'autre part, dans Les Coréens, l'échange d'uniformes a un effet ironique similaire : deux membres du peloton français retrouvent trois cadavres nordcoréens. Ils échangent leurs vêtements avec ceux des guerriers morts. Déguisés en soldats coréens, ils rencontrent Ir-Won, le villageois qui a collaboré avec les Américains. Les deux Français l'attirent. En tant que Coréens du Nord, ils font ce qui se doit ; le punissant comme traître qui a collaboré avec l'ennemi. Puis ils réalisent qu'Ir-Won, avec son sac à dos sur son épaule, n'a pas l'air d'un soldat, alors ils décident de lui donner l'uniforme du troisième coréen mort. Ainsi, les rôles sont intervertis par ces déguisements. L'échange d'uniformes nous rappelle à quel point un personnage peut changer de nationalité. Vinaver utilise cette farce pour traiter de sérieuses questions sur le rôle de l'armée française d'outre-mer et l'absurdité de certaines politiques gouvernementales ${ }^{34}$. Vinaver établit un contraste dynamique entre le comique et le macabre. Belair, demeuré évanoui à côté du cadavre de Toung, se réveille et lui adresse ces paroles: "Tu en as de la chance, Sing Sing, vieux frère. [...] Depuis ce matin que tu n'es plus sur la terre mais dedans, ça commence à aller mieux, non? ${ }^{35}$ »

\footnotetext{
${ }^{32}$ ID, (1955), « Les Mythes de la Grèce ancienne, une marche d'approche », in Critique, mars, p.199.

33 «Toute l'œuvre de Michel Vinaver s'applique à puiser ses sujets dans l'actualité. »

MORTIER D., Celui qui dit oui, celui qui dit non ou la réception de Brecht en France (1945-1956), ),

Paris-Genève, Champion-Slatkine p.287.

34 cf. GUREWITCH M., (1975), Comedy : the Irrational Vision, NYC, Cornel U.P., p.234.

${ }^{35}$ VINAVER M., (1986), op.cit., p.83/84.
} 
Sous le contraste apparent entre la comédie et la gravité des situations se cache tout l'éclat de l'art de Vinaver, où la théâtralité gagne à côtoyer le danger. Les soldats déguisés en uniformes ennemis sont en grave danger émoussé par les ébats comiques. Ainsi l'idée de patrie se trouve compromise par la comédie farcesque: «Le rire est l'instrument par lequel nous nous purgeons du scandale et de la contradiction dévoilée, par lequel l'incongruité est acceptée et dépassée. ${ }^{36}{ }{ }^{2}$

Les deux pièces tentent d'impliquer les spectateurs dans un environnement politique familier, les invitant à reconsidérer leurs pensées et réflexions politiques: "l'invitation à un changement immédiatement réel ${ }^{37}$." Si Vinaver tolère le théâtre subversif de Jarry c'est qu'il est un " théâtre à nature provocatrice ${ }^{38} \gg$ qui secoue l'opinion du spectateur aussi bien au théâtre que dans la vie. Dans un compte-rendu d'Ubu Roi, Vinaver semble spécialement attiré par cette pièce, il note ses qualités puissamment troublantes: "Ubu Roi détraque le système de nos idées reçues, oblige à une révolution dans la fonction de la dramaturgie. ${ }^{39}$ " Jarry emprunte l'intrigue et le complot du théâtre politique historique de Shakespeare mais sa pièce est une satire politique contemporaine de la période de la Troisième République. Il présente aussi d'une manière provocante Ubu (ex-roi d'Aragon), qui s'empare de la couronne par le meurtre et maintient le pouvoir à travers une répression vicieuse. Le rêve d'Ubu de posséder un parapluie rappelle Adolphe Thiers, président au cours de la $3^{\text {ème }}$ République, lors des négociations avec les Allemands suite à la guerre Franco-prussienne, «toujours caricaturé à l'époque avec un parapluie $e^{40} \gg$. Celui-ci organise la répression sauvage et violente de la Commune de Paris en Mai 1871. Ainsi, la pièce de Jarry offre une description sinistre des motivations du gouvernement conservateur qui s'oppose à tout changement et insiste à perpétuer un ordre révolu. Pour lui, cette pièce provoque un rire amer qui n'est ni de l'amusement ni de la bonhomie : «c'est le rire qui correspond à la découverte de la situation "inénarrable » de l'homme dans le monde. ${ }^{41} »$

La même technique a été mise en œuvre par Vinaver dans la pièce, 11 Septembre 2001, où le rire ne représente ni une échappatoire, ni une dénonciation coléreuse de la société, mais plutôt un clin d'œil, un rappel pour éveiller les esprits, il «pointe ainsi [...], l'irréductible inadéquation de l'homme et $d u$ monde ${ }^{42} »$. Par cet événement qui a secoué le monde entier, avec ses énormes impacts atteignant tout le système politique et social des démocraties

${ }^{36}$ ID, (1998), op.cit., p.34.

37 Ibid, p.36.

${ }^{38}$ ELSTOB K., (1992), op.cit., p. 22.

${ }^{39}$ VINAVER M., (1998), op.cit., p.35.

${ }^{40}$ DAMERVAL G., (1984), Ubu Roi : La bombe comique de 1896, Paris, A.G. Nizet, p.96.

${ }^{41}$ VINAVER M., (1998), op.cit., p.34.

42 THOMASSEAU J-M., (2006), « L'ouï-dire et l'inouï » in Revue Europe, (avril 2006, n 924,

Numéro spécial Michel Vinaver), p.4. 
occidentales, Vinaver cherche à troubler l'ordre des choses. En juxtaposant des images, des objets ou des événements, il force le spectateur à rester alerte, à tisser activement les liens entre les images ou les événements. Il dresse devant lui les maitres du monde sous forme de deux « dieux » en conflit, celui de G. W. Bush et celui d'Oussama Ben Laden : "Ben Laden: Voici l'Amérique frappée par Dieu tout puissant en un de ses organes vitaux [...] Bush: De ce forfait les talibans peuvent s'attendre à payer le prix les terroristes auront beau s'enfoncer au plus profond de leurs cachettes souterraines

Ben Laden: Alors le monde entier est entré dans la plus grande panique les infidèles suivis des hypocrites [...] Bush : face à la nouvelle menace d'aujourd'hui la seule façon de poursuivre la paix Est de poursuivre ceux qui la menacent [...] Ben Laden : Je jure devant Dieu [...] Que l'Amérique ne vivra pas en paix [...] Tant que l'armée des infidèles n'aura pas quitté jusqu'au dernier la terre de Mahomet ${ }^{43}$ "

Les parties divergentes n'entrent pas en opposition directe; elles se tiennent côte à côte, nous invitant à nouer des liens entre eux, modifiant le concept d'une nation unifiée. Dans Les Coréens, les mondes restent déconnectés: Les forces qui se manifestent ne sont pas résolues dans un dénouement comique ou tragique, les microcosmes de Vinaver admettent des contradictions et ne les atténuent pas. Le bataillon français perdu dans les rizières est opposé à la population du village coréen. Le raisonnement patriotique familier de la troupe française contraste avec la détermination des villageois à survivre malgré la guerre: les uns réagissent comme une bouche pleine de slogans vides de sens, alors que les autres sont bien enracinés dans leur sol natal. Ces contradictions incitent le public à reconsidérer les fondements du patriotisme français.

Chacun reste libre de choisir le clan qui lui convient. Mais pour la majorité, Vinaver le souligne, le salut réside probablement dans l'incrédulité ! ${ }^{44}$

Dans les deux pièces, l'auteur confronte l'audience avec des aspects diaboliques de leur propre société mais sous le couvert d'une ironie volatile, "au niveau moléculaire, celui des répliques et des liens qui se créent entre elles ${ }^{45}$ », produisant des effets de sens que le lecteur essaye de repérer. Le "Qu'est-ce qu'ils allaient faire en Corée? ", proféré par Beaugeron aux Coréens, compromet la politique de la France d'outre-mer. Les raccourcis que

\footnotetext{
${ }^{43}$ VINAVER M., (2002), 11 Septembre 2001, (2002). Paris, L’Arche, pp. 61-67.

${ }^{44}$ Babelio https://www.babelio.com/livres/Vinaver-11-septembre-2001/175909 consulté 28/10/2017.

${ }^{45}$ RYNGAERT J-P., (2006), «L'Ironie contre l'esprit de sérieux. Présence du comique chez Michel Vinaver » in Revue Europe, (avril 2006, n 924, Numéro spécial Michel Vinaver), p.20.
} 
profère Bush dans 11 Septembre 2001, pour satisfaire toutes les parties en litige prêtent non seulement à rire mais lui font perdre toute crédibilité aux yeux du monde entier. Il dit : En même temps que nous frapperons les cibles militaires nous lâcherons de la nourriture ${ }^{46}$ "; ou encore : «Il est vital de continuer à consommer/ de continuer à acheter ${ }^{47}{ }$ ”

Quant à Rumsfeld, appelé à rassurer les citoyens, il utilise, en parlant de l'ennemi, une image : "A la fin ils s'effondreront de l'intérieur/ c'est cela qui constituera la victoire ${ }^{48}$ " alors que ce sont effectivement les deux tours qui venaient de s'effondrer de l'intérieur. L'originalité de la pièce tient dans le fait de fixer l'événement par le choc des voix, l'entrecroisement des paroles, issues de diverses sources journalistiques, les répons de discours, le tout commenté à l'antique par un chœur. ${ }^{49}$ En regardant la pièce, on reconnaît les échecs, les maladresses et les faiblesses des gouverneurs des superpuissances. L'auteur essaye d'éveiller ce type de conscience chez le spectateur en mettant sur scène ce que celui-ci s'efforce de ne pas voir. ${ }^{50}$ En effet, Vinaver salue cette technique surréaliste, utilisée par Jarry, d'associer librement des images dissociées qui contrarient le monde bourgeois par le choc de la logique habituelle et les associations mentales :

"Expression de notre temps par l'image explosive qu'elle nous renvoie de nos contradictions, Ubu Roi inaugure aussi un théâtre de notre temps sous l'aspect de la structure formelle. En rupture formelle avec les modes et les genres du théâtre traditionnel, Ubu Roi, superbement fait table rase de la continuité chronologique psychologique et simplement logique de l'action, vestige d'un règne de la Raison que le respect des classiques et le confort d'un alibi ont perpétué dans notre théâtre jusqu'à ce jour. ${ }^{51}$ " Il revendique de «bousculer le spectateur dans son ordre établi » sans pourtant renverser «l'ordre des mots et celui des idées reçues d'une façon directement provocatrice. ${ }^{52} \gg$

\section{Ecriture du quotidien:}

Dans son ouvrage, Ecrits sur le Théâtre, Vinaver, souligne l'importance d'une perspective contemporaine d'un monde polyvalent et d'un besoin de sonder et de saisir l'actualité brute de la vie quotidienne. Pour lui, les images doivent se superposer comme dans le montage cinématographique afin de réfléchir la vie

\footnotetext{
${ }^{46}$ VINAVER M., (2002), op.cit. , p.63.

${ }^{47}$ Ibid, p.53/54.

${ }^{48}$ Ibid, p.33.

${ }^{49}$ Académie de Paris, Pièce démontée, Théâtre de la ville, $\mathrm{n}^{\circ} 1$ « Les dossiers pédagogiques « Théâtre » et «Arts du cirque » du réseau SCEREN en partenariat avec le Théâtre de la Ville, pp.1-29, http://crdp.ac-paris.fr/piece-demontee/pdf/11-sept_total.pdf consulté 2/3/2018, p.1.

50 cf. VINAVER M., (1998), op.cit., p.36.

${ }^{51}$ Ibid, p.32.

${ }^{52}$ Ibid, p.35.
} 
réelle.

«Je me dis aujourd'hui que si le fait de traiter ici et maintenant au théâtre suscite des résistances, il faut quand même le faire, et qu'il faut chercher à le faire mieux, c'est-à-dire en allant vers l'accessibilité la plus grande et l'agrément le plus vif, et que ceci peut se faire sans concession aucune; je me dis qu'une dramaturgie du présent, à certaines conditions, peut activer des forces latentes que ni la télévision ni aucune autre forme de divertissement ne saurait mettre en éveil. ${ }^{53} »$

Homme d'affaires et homme de théâtre, Vinaver s'appuie sur ses propres expériences de la politique aux facettes multiples, de l'économie et de la culture. Depuis ses débuts, il découvre une nouvelle forme de performance théâtrale, où il tente de réintroduire des thèmes de la vie quotidienne avec la solennité et la force sémantique ${ }^{54}$ qui avaient cédé la place aux événements éclatants. Il crée un théâtre aux résonnances plurielles : du mythe grec ancien à la structure théâtrale brechtienne, du surréaliste au banal. Vinaver se concentre sur ces ambiguïtés. Il ne se propose pas de donner une interprétation politique, mais, par son portrait de la vie quotidienne, il nous fait part, avec une ironie inquiétante, de la politique de la vie quotidienne. Il trouve que le théâtre n'est pas une simple représentation abstraite mais une force qui émerge de « l'actualité », à la quelle il doit s'engager : "raconter le tissu de l'histoire » et "laisser les ponts se faire, les jugements se former ». Pour lui, c'est la véritable essence d' "une action politique qui soit en prise sur la situation réelle ${ }^{55}$ » actuelle. La première pièce, Les Coréens, était à l'origine intitulée Aujourd'hui. D'une production à l'autre, la pièce change de titre: d'Aujourd'hui elle devient Aujourd'hui ou les Coréens, puis les Coréens tout court. Bien que le thème de la pièce soit la guerre de Corée, le titre original inaugure une conception théâtrale du jour présent, "le présent immédiat, l'histoire, est à la fois la matière même du texte et le terreau où les personnages puisent à chaque instant un vouloir-vivre renouvele..$^{56}$ "Notre attention est attirée vers une certaine banalité quotidienne ou vers un groupe de gens ordinaires distingués uniquement par leur espace commun ou leur nationalité commune. Etant entouré de troupes Coréennes, qu'il ne parvient pas à voir, Exaxerguès, soldat du bataillon français, est effrayé par la confusion de la guerre dans la jungle. Pour lui, la guerre doit consister en un champ plat, avec des ennemis se faisant face de chaque côté du champ, "pas ces putains de broussailles dans lesquelles on tourne en rond et on ne voit jamais rien. ${ }^{57}{ }$ Son raisonnement

\footnotetext{
53 ID, (1989), « Ici et maintenant », in $\boldsymbol{L}$ 'Art du théâtre, octobre, p. 41.

${ }^{54}$ ID, (1998), op.cit., «Sur une représentation d'Hamlet à Annecy », p.23.

${ }^{55} \mathrm{Ibid}, \mathrm{p} .107$.

${ }^{56}$ UBERSFELD A., (1989), Vinaver dramaturge, Paris, Librairie théâtrale, p.13.

${ }^{57}$ VINAVER M., (1986), op.cit., p.94.
} 
compromet la décision de guerre prise par son pays, qu'il résume en remettant en question la politique nationale, "s'il ya une autre guerre, d'abord je prendrai mes renseignements. ${ }^{58} \gg$ Vinaver ne prétend guère tirer de conclusions à travers ces éléments divergents du dialogue qui ont pour seul intention d'interroger ou de pointer certains agissements du pouvoir. Pour lui, c'est une forme rudimentaire de montage, puisque seuls deux éléments sont en contraste : «La mise en regard de deux mondes réfractaires l'un à l'autre, les soldats du corps expéditionnaire français et le village coréen. L'efficacité de la pièce tient beaucoup au frottement entre deux langages fortement contrastés, celui des soldats, celui des villageois. ${ }^{59}{ }^{\prime}$

Dans ses pièces, Vinaver dépeint des personnes qui, comme lui-même, «doivent avoir du mal à pénétrer le territoire de la vie quotidienne. » Il transforme la vie quotidienne en un monde ludique et fragmenté, qui ébranle l'ordre établi, non pas en présentant la révolution sur scène, mais en reconstruisant de nombreuses interprétations de l'ordre social. Les pièces politiques qui en résultent tentent de provoquer une prise de conscience chez le public, mais en évitant les stéréotypes des pièces qui tentent de montrer comment avoir une révolution. Utilisant la contradiction et la discontinuité, «ils démontrent plutôt que de discuter des vies brisées qu'ils choisissent de dramatiser. ${ }^{60} \gg$ Sa technique dramatique consiste à porter à la scène une réalité fragmentée d'une vie quotidienne «où les motivations personnelles des individus et le comportement des organismes publics semblent être hors de contrôle. ${ }^{61}{ }^{\prime}$ Dans ses pièces, il utilise un type de composition qui représente des interprétations parallèles d'une même réalité reposant sur un montage d'intrigues, de scènes épisodiques, de dialogues fragmentés, et parfois d'espace scénique, qui achèvent de bouleverser l'ordre établi. Il rejette l'idée de concevoir la vie des individus comme une série d'événements bien ordonnés; mais une suite d'éléments incongrus dont la juxtaposition affirme l'impossibilité de parvenir à une vue mondiale unifiée et renvoie à l'idée d'un monde en discordance. Le collage de fragments qui se heurtent et résonnent entre eux «produit des inconsistances ironiques entre l'horizon d'attente et le réel. ${ }^{62} \gg \mathrm{Il}$ a pour valeur de rapprocher la politique du gouvernement de la routine quotidienne, qui montre le citoyen pitoyablement broyé sous le poids du quotidien déformé par un pouvoir écrasant, cause de l'exploitation, de l'aliénation, du terrorisme comme toutes les autres iniquités du système politique. Ainsi, un circuit traversé par «un courant sémantique ${ }^{63} »$ est créé,

${ }^{58}$ Ibidem

${ }^{59}$ ID, (1998), op.cit., p.288.

${ }^{60}$ BRADBY D., (1984), op.cit., p. 240.

${ }^{61}$ Ibidem

${ }^{62}$ DIAZ Sylvain et NOEL Anne-Sophie, «Les Troyennes, tragédie new-yorkaise ?», Agôn[En ligne], Dossiers, (2011) HS n 1 : Mettre en scène l'événement, 11 septembre 2001 de Michel Vinaver, mis à jour le : 23/12/2011, URL : http://agon.ens-lyon.fr/index.php?id=1782. consulté le 17/9/2017.

${ }^{63}$ VINAVER M., (1979), Les Travaux et les Jours, Paris, l'Arche, p.75. 
ayant pour objectif de dresser une nouvelle manière de voir et de s'exprimer. Pour réaliser une harmonie multilatérale, Vinaver a recours à deux termes qui peuvent aider à définir son théâtre par un lexique spécifique qui oscille entre la "densité » et l' "entrelacs ${ }^{64}$ ", dont il développe les significations dans «une écriture $d u$ quotidien ${ }^{65} »$. La densité étant la matière poétique tirée de "l'indistinction quotidienne ${ }^{66}$ » qui acquiert une extrême densité ou « tension poétique (qui) dépend de la richesse des connections qui se sont faites. ${ }^{67}{ }_{\gg}$ Ces deux techniques caractérisent l'esthétique dramaturgique de Vinaver d'une manière analytique, soit au niveau «de l'agencement des sons, des mots, des phrases, des répliques ${ }^{68} \gg$, soit, sur une plus grande échelle, porter au clair l'assemblage de ses pièces entre elles.

Dans Les Coréens, Vinaver présente deux mondes diamétralement opposés. La guerre s'infiltre dans la vie quotidienne des villageois coréens. Elle touche aux menus détails depuis la nourriture et l'approvisionnement jusqu'au mariage des enfants, depuis la reconstruction des chaumières dévastées par les raids des ennemis jusqu'au départ des jeunes enrôlés dans la résistance. De l'autre côté de l'océan, en France, dans la patrie lointaine, personne n'interagit avec les soldats français perdus dans les forêts tropicales. Parmi eux, le lexique guerrier est remplacé par leurs rêves et leurs souvenirs qui se déroulent ailleurs:

"Beaugeron: Il avait une bagnole chez lui, un vrai engin de sort, il faisait du cent quatre-vingts. Il avait une femme et quatre enfants. Il s'est engagé parce que sa femme ne voulait plus qu'il fasse de

l'automobile.

\section{Exaxerguès: Qui, Belair?}

Bonassier: Non, Rossetti. Belair c'était un comptable.

Lhomme: Non, c'était un représentant.

Beaugeron: D'abord il a été un comptable, et puis représentant. ${ }^{69}{ }$

L'opposition distincte entre ces deux mondes demeure du début à la fin de la pièce. Elle est symbolisée par la mort versus la vie. D'ailleurs la mort, présente et éminente dans les deux pièces sous différentes formes, surtout à la guerre ou dans l'attentat, semble constitutive du système de vie, de l'existence elle-même. La mort ne paraît pas témoigner du mal mais simplement du négatif. Exaxerguès: J'en reviens pas, moi, qu'il soit passé dans les roses.

Lhomme: Belair.

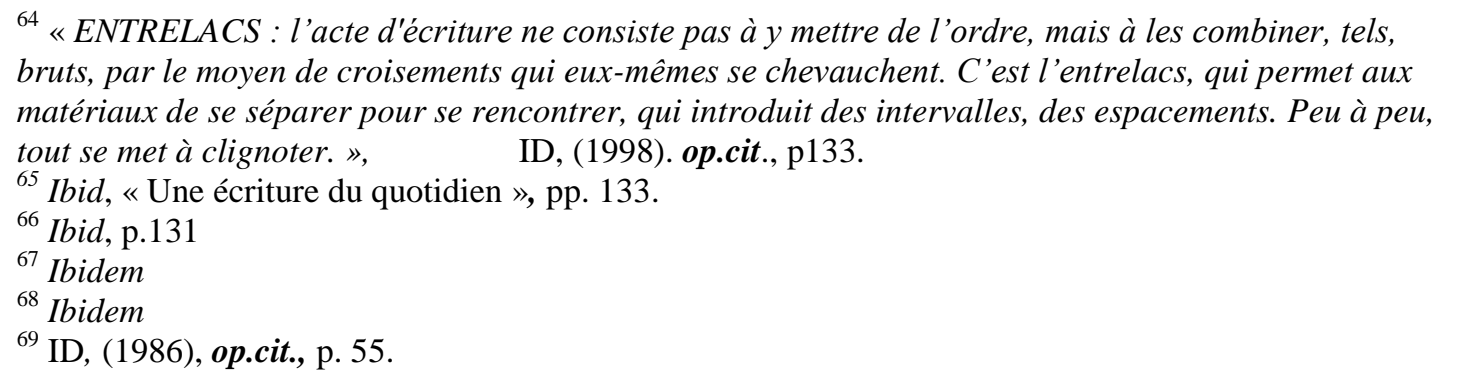


Lhorizon: Il est passé dans les roses? (...)

Exaxerguès: Eh bien, j'aurais parié n'importe quoi qu'il serait le dernier.

Beaugeron: De qui tu causes? Et le dernier à quoi?

Exaxerguès: Belair. J'aurais parié qu'on serait tous passés avant lui. Comme au théâtre quand on sait d'avance ce qui va arriver. ${ }^{70}{ }{ }$

C'est pour cette raison que Vinaver ne distingue pas la mort physique de celle qui se produit constamment dans le tissu social. Pour le public, cependant, les images des deux mondes sont appelées constamment à interagir. Nous sommes appelée à voir alternativement les Coréens puis les Français, selon des tableaux juxtaposés qui nous obligent à les comparer sous une lumière nouvelle et plus complexe. Les villageois coréens sont véritablement démocrates : ils sont soit pro-communistes, soit pro-capitalistes, mais les besoins de leur communauté les unissent. Alors que les soldats français ne sont unis que par devoir : les ordres militaires leur donnent un but commun, mais leur maladresse à exécuter les ordres ébranle leur but. Dans Les Coréens, le caporal français, Belair, considéré comme mort à la suite d'une bataille, son régiment se trouve abandonné sans chef ; alors les soldats se choisissent un autre chef, tentant d'exécuter ses ordres dans une veule obéissance. C'est là qu'intervient le refus, qui constitue le cœur du théâtre de Vinaver. A l'opposé de cette obéissance, Belair déserte la troupe française pour se rendre au village coréen où il découvre la chaleur de la communauté humaine, incarnée dans le geste d'amour de la petite fille qui lui donne le caillou permettant « de ne pas mourir $^{71} »:$ : je ne sais pas demain ce qui se passera, dit-il, mais aujourd'hui je vais vous dire, j'ai envie de rester ici, jusqu'à ce que cette guerre soit finie, et quand elle sera finie, de rester encore ici. ${ }^{72} \gg$

Chacun des Coréens a sa propre vision de la survie dans des conditions de guerre. Certains d'entre eux soutiennent l'armée du Nord et d'autres l'armée du Sud. Ir-Won a même accepté les aliments de l'ennemi américain; le fils de Hun-Tan, Ten, rejoignit l'armée du Sud ; il n'avait pas le choix quand Syngman Rhee a décrété la mobilisation; Tuong, le frère de Wen-Ta, a été arrêté par les soldats français pour avoir planté les mines en vue de faire exploser les soldats de l'armée du Sud. La guerre affecte tout en Corée même la conversation la plus banale: «Lin-Huai (la mère de Wen-Ta) : Tu sais je pense que Wou-Lon devrait épouser le petit Kim. Il la regarde depuis bien longtemps, et maintenant que Ten est mort. ${ }^{73}$ "

La mention de Ten rappelle que l'horreur de la guerre, produisant mort et destruction dans la communauté, est à portée de main.

\footnotetext{
${ }^{70}$ Ibid, p.56

${ }^{71}$ UBERSFELD A., (2006), "Vinaver et le Coeur "in Revue Europe, (avril 2006, n 924, Numéro spécial Michel Vinaver), p.103.

${ }^{72}$ VINAVER M., (1986), op.cit., p.119.

${ }^{73}$ Ibid, p.61.
} 


\section{Le quotidien, voix des opprimés :}

L'auteur présente des personnages brisés par les institutions. Les personnages ne sont pas conscients de leur perte, mais le spectateur reconnaît sa propre condition dans celle de ces personnages. Ces derniers nous rendent compte des problèmes que nous tentons d'omettre, au cours de notre routine quotidienne. Ses personnages sont souvent ordinaires, parfois ouvriers, leurs problèmes apportent une nouvelle dimension à l'interprétation théâtrale de la politique. Il accorde une place de choix à la parole et au témoignage des victimes, des simples individus et du chœur, affirmant que la seule position admise est celle qui consiste à " $s$ 'orienter $d u$ côté $d u$ petit contre le grand, $d u$ faible contre le fort. ${ }^{74}{ }$ V Vinaver dramatise la vie quotidienne de ceux dont les voix n'avaient pas été entendues auparavant, faisant, ainsi écho à l'un des thèmes de prédilection de Jean Genet qui donne la parole aux personnes exclues à cause de la couleur de leur peau (Les Nègres), ou de leur condition sociale (Les Bonnes), dont l'effet est indéniable pour faire entendre la voix des femmes écrasées d'une nouvelle manière. Mais Vinaver se distingue de Genet par la création de personnages ordinaires et assez simples, donc très différents des personnages de ce dernier ayant un dialogue poétique et riche.

Dans d'autres contextes, les actions des personnages ne correspondent pas à leurs rôles et sapent certaines hypothèses fondamentales de la patrie : Belair, soldat français de Les Coréens, n'agit pas par ferveur patriotique ou par volonté idéologique. Au cours de ses rencontres avec les villageois coréens, il affronte des opinions anti-communistes, qui justifient l'envoi de troupes françaises en Corée. Mais en creusant la terre à la recherche d'un pot de confiture à donner à Brooks, son compagnon mourant de la Première Division américaine des forces expéditionnaires des Nations Unies en Corée, il trouve une boule de bois et se souvient des enfants coréens qui devaient jouer dans la rue avec ce type de balle. Ces "sauvages" n'ont pas encore connu le caoutchouc, pense-t-il. Mais quelques secondes plus tard, il rencontre Wen-Ta, une coréenne de huit ans et tous les deux se mirent à creuser une tombe pour Brooks. Wen-Ta explique que son frère a été emmené pour être abattu, parce qu'il plantait des mines tout au bord de la route. "Qui l'a tué?" demande Belair, "les soldats blancs", répondelle. Comme si Belair était exclu de cette légion. Leur amitié naissante défie les idéologies conflictuelles. Wen-Ta le ramène dans son village, dont les habitants le nourrissent et s'occupent de lui. L'accueil du caporal dans la communauté villageoise pulvérise l'unité de la force française d'outre-mer. Pourtant, Belair ne dénonce pas l'idéal de la patrie mais il amène le public à un niveau différent de compréhension de l'engagement patriotique dans la guerre. Il clarifie l'opposition entre l'homme et le métal qui fait partie intégrante de la guerre :

74 DARGE F., (2009), « Michel Vinaver, dramaturge du réel », propos recueillis par Fabienne Darge, in Le Monde 2, n 258, 24-30 janvier, p. 25. 
"Le métal ça surprend toujours, ce que ça peut faire quand ça entre dans le corps d'un homme. T'as des liquides qui coulent dans les tubes flexibles et transparents, et des pâtes et des matières molles. ${ }^{75}$ » Ses mots trouvent un écho dans les attitudes de son peloton. Ils révèlent la contradiction entre l'être humain jouant au soldat et la nature violente de ce rôle. Belair est un homme pensif, incertain, qui se trouve être un soldat. Les forces françaises ne sont qu'un groupe de soldats faisant ce qu'on leur dit; les soldats français ne sont ni des héros ni des anti-héros, ce sont des «mercenaires qui se livrent à une guerre insensée ${ }^{76}{ }_{\gg}$. Se trouvant isolé de son peloton, Belair se laisse absorber dans la communauté villageoise coréenne, où il demeure définitivement. Ses activités n'impliquent pas un changement révolutionnaire, mais un mouvement vers la simplicité, avec des gens qui vivent dans des maisons entourées de voisins. La désertion de Belair n'est pas la conséquence d'une position anti-impérialiste, mais d'un retour à la chaleur de la vie simple des villageois. ${ }^{77}$

Parallèlement, l'action de Vinaver est de trouver une voix pour ceux qui, auparavant, n'ont pas été entendus sur scène à cause de leur classe. Il est vrai que ses pièces ne se concentrent pas directement sur les questions raciales, elles reflètent plutôt la tendance du théâtre à dramatiser l'oppression afin d'inciter le public à reconsidérer le système politique. En outre, l'histoire jaillit du fond de la dramatisation de la vie quotidienne, provoquant «de petits éclats de conscience au ras du magma. ${ }^{78}$ »

Dans 11 Septembre 2001, le monologue, clôturant la pièce, d'une jeune femme ayant échappé aux attentats, pourrait témoigner de cette volonté de faire parler les impuissants : comme tout autre spectateur, elle apparait hantée par l'éventualité de ce qui aurait pu arriver :

"Bien sûr oui bien sûr il aurait fallu que j'y sois au bureau

Dieu merci Tommy a eu son indigestion il a vomi toute la nuit

J'étais vraiment inquiète Paul me dit tu pourrais pour une fois rester à la maison prendre un peu de repos [...]

Il y aura toujours un meeting par-ci un meeting par-là chérie Paul me dit M. Gainsborough comprendra regarde-toi dans la glace t'as une mine de déterrée je te jure

Bon je dis

Et maintenant et maintenant et maintenant ${ }^{79}{ }$

75 VINAVER M., (1986), op.cit., p.47.

${ }^{76}$ LAMONT R., (1988), op.cit., p.383.

77 cf. BARTHES R., « Préface » in Théâtre Complet I de Michel Vinaver, p.37.

${ }^{78}$ VINAVER M., (1998), op.cit., p.308.

${ }^{79}$ ID, (2002), op.cit., p.181. 
En somme, le quotidien est crucial pour la compréhension de l'écriture de Vinaver.

«Le théâtre du quotidien » fut un nom donné à un groupe d'écrivains à savoir : Michel Vinaver, Michel Deutsch, René Kalisky, Jean-Paul Wenzel, qui confrontèrent leur audience par :

«des scènes fragmentaires montrant des personnages ordinaires, souvent incapables d'articuler, dans des situations très ordinaires, présentées néanmoins dans un style de réalisme accru. Derrière la banalité des situations, la violence cachée des structures sociales contemporaines a émergé avec une grande force. ${ }^{80}{ } \gg$

Ces dramaturges explorent la notion de « quotidien » à travers une collection variée de personnages ordinaires, anonymes qui ont du mal à comprendre le monde dans lequel ils vivent. Ils dépeignent des individus condamnés à se rebeller contre des institutions qui ne cessent de se renforcer au fur et à mesure que la communauté se brise. Ainsi, ils illustrent l'affirmation de Vinaver selon laquelle nous vivons en communion avec un système qui nous détruit. Sur la scène, les pièces le signalent en présentant des personnages dans des situations marginales du système capitaliste, faisant ainsi entendre «la parole de ceux qui la manient mal pour la simple raison que, sur la scène de l'Histoire, ils ne l'ont pas encore prise. ${ }^{81} \gg$ Ces écrivains accordent une voix à ceux qui ne furent pas écoutés auparavant, rejoignant les échos motivant l'initiative d'Armand Gatti qui, à défaut de scène, présentait ses pièces dans un garage, et le théâtre collectif du Soleil mettant centralement en scène les classes opprimées.

Dans 11 Septembre 2001, Vinaver juxtapose l'incident terroriste à la vie quotidienne des habitants de Manhattan. Si l'explosion des deux tours est motivée par les enjeux de la politique internationale entre Occident et MoyenOrient, l'impact des attentats retombe sur la population newyorkaise (le politique). Ces changements alternatifs prouvent que l'intérêt de l'auteur est essentiellement porté sur l'immédiat, le quotidien. Ces deux éléments sont juxtaposés par l'introduction des personnages ordinaires engagés dans des situations critiques. D'une part, les réactions vis-à-vis de l'attentat sont transmises à travers plusieurs voix anonymes ou employés et ouvriers ordinaires qui représentent la main d'œuvre principale des deux tours et la véritable population lésée ; d'autre part, le spectateur est témoin de la vie quotidienne des habitants moyens de la cité de New York. En rapportant deux aspects du quotidien dans un contexte politique défini, l'auteur attire l'attention sur ce qui lie la politique d'usage individuel au contexte plus vaste, celui de la politique extérieure. Puisés dans l'actualité, ses sujets tiennent compte des situations, des

\footnotetext{
${ }^{80}$ BRADBY D., (1984), op.cit., p.235.

${ }^{81}$ SANDIER G.,(1982), Compte-rendu de Dissident, il va sans dire et Nina, c'est autre chose,

Théâtre en crise, Grenoble, La Pensée Sauvage, p. 243.
} 
émotions et des identités quotidiennes ; c'est un théâtre qui invite son public à prendre position dans le tissu quotidien d'une nation « en déliquescence ». En en percevant les fissures, il revient au spectateur de soulever des interrogations.

\section{Paradoxe du quotidien et du mythique :}

Vinaver est un habile magicien à communiquer par le théâtre. Ce dernier aura pour tâche d'éveiller les consciences et de les habiliter en vue de communiquer une nouvelle vision de la vie. Il prétend que ce médium ouvre un passage vers "une configuration nouvelle des idées, des sentiments, des valeurs. De forcer la porte à un comportement non encore imaginé. ${ }^{82}{ }^{\star}$ Pour lui, le théâtre représente une part intégrale de la vie sociale, ayant la même importance que l'eau, le pain, le feu et l'abri. ${ }^{83}$ Son concept de théâtralité subit la transcendance du théâtre grec classique. La réinterprétation de ce dernier consiste à créer une conscience nouvelle à travers les pièces qui attirent l'audience par quelques scènes familières, tout en jetant une lueur originale sur ce qu'on considère comme un fait accompli dans notre vie quotidienne.

Or, son intérêt pour le quotidien semble en discordance avec celui qu'il accorde au théâtre grec. Il importe donc de dresser des analogies entre 11 Septembre 2001 et le théâtre classique pour voir comment le théâtre de Vinaver fait écho à certaines de ses composantes et comment il les amalgame pour produire une dramaturgie qui sonde la situation politique et économique. Vinaver fait largement usage des outils du théâtre antique. Il emprunte « le chœur » qu'il a su ressusciter dans nombre de ses pièces pour lui servir de voix de la conscience qui soulève des questions politiques en dévoilant les voix inouïes des personnages de l'histoire passée et présente. Cela contrebalance les voix qui sont entendues sur la scène politique. En réunissant ces voix de manière à ne pas désigner une seule voix comme celle qui monopolise la vérité, l'auteur nous laisse penser à l'état de choses. Il nous encourage à mettre l'ordre établi dans une nouvelle perspective. Les passages choraux sont particulièrement fréquents dans 11 Septembre 2001. De surcroît, par souci de vraisemblance, ces passages sont débités en anglais traduisant, dans une sorte d'hallucination effarée et décousue, la consternation de la population newyorkaise ordinaire. Lors de l'attentat, le chœur profère une longue déploration du sort du commun des humains, confrontés à un événement exceptionnellement tragique : «Hi Jacked / Hi Jacked Jets Jackety Jets / Hijacked Jets / Hi Jets Hit Trade / World Weird / Worderly Trade / Pentagon / Twin Towers /

${ }^{82}$ VINAVER M., (1998), op.cit, p.35.
83 cf. Ibid, p.115. 
Falling Down Falling Down Falling / Gone /

The Twin Towers Are Falling Down Falling Down Falling Down ${ }^{84} \gg$

Plus tard, à chaque mesure prise par le pouvoir correspond un refrain satirique du chœur. Au discours de G.W. Bush encourageant les financiers à poursuivre sans interruption leurs placements bancaires, leurs achats d'actions à Wall Street malgré les éventuels dégâts, à ne pas se résigner ni reculer devant les incidents terroristes ; le chœur gémit :

«Keep Desires Afloat [...] / For This is War Prepare /To Suffer Hardships / Consent Sacrifices ${ }^{85}{ }^{\prime}$

L'intervention épisodique du journaliste, combinée au chœur sert également à conférer à la pièce la forme rythmique voire musicale d'une « cantate » à trois voix ou d'un « oratorio » ${ }^{86}$, qui fait office de tombeau. Cela sert de service funéraire pour les victimes des attentats. Les lamentations se répondent. Le chœur effaré s'affolle :

«Paths / Of Terror a Nation / Mourns ${ }^{87}$ »

Et le journaliste d'affirmer la catastrophe :

«Ici où je me tiens ici / Ground Zero je vois / Des scènes inoü̈es de chaos et de peur /

Certains sont vivants d'autres morts certains étaient vivants ${ }^{88} \gg$

D’autre part, 11 Septembre 2001 présente un substrat quasi antique sous l'apparence moderne de la forme aussi bien de l'écriture que de la mise en scène. Michel Vinaver y décrit George W. Bush et Ousama Ben Laden, de manière assez inattendue, comme des «dieux» antiques, «Il introduit son lecteur-spectateur dans le monde des dieux antiques, de la fatalité du sort qui $s$ 'abat sur les êtres. ${ }^{89} \gg$ Aussi la détermination divine de ces deux personnages se trouve-t-elle renforcée par leurs propos croisés fortement religieux à la fin de la pièce, au point que leur action ne semble motivée que par leur foi ${ }^{90}$.

«Bush : Bonjour sur mes ordres l'armée des États-Unis a commencé des frappes

Ben Laden : Voici l'Amérique frappée par Dieu tout puissant en un de ses organes vitaux

Bush : Nous sommes soutenus dans cette opération par la volonté collective du monde

entier

\footnotetext{
${ }^{84} \mathrm{ID},(2002)$, op.cit., p.23/24.

${ }^{85}$ Ibid, p.55.

${ }^{86}$ Ibid, « Note liminaire », p. 9.

${ }^{87}$ Ibid, p.28/29.

${ }^{88}$ Ibid, p. 29

${ }^{89}$ Ibid, «Notes manuscrites », p. 286.

${ }^{90}$ cf. ibid, pp. 179-181.
} 
Ben Laden: De sorte que ses plus grands édifices sont pulvérisés par la grâce de Dieu à

qui va notre gratitude $[\ldots]$

Bush : Et nous sommes les amis de près d'un milliard de gens qui pratiquent la religion

islamique dans le monde entier

Ben Laden: L'hypocrisie a levé haut la tête lamentant la perte de ces assassins qui ont

joué avec le sang l'honneur et les sacrements des musulmans

Bush : Nous sommes une nation pacifique

Ben Laden: Ils ont soutenu le boucher contre sa victime l'oppresseur contre l'enfant

innocent

Bush : Face à la nouvelle menace d'aujourd'hui la seule façon de poursuivre la paix

Ben Laden : Le vent du changement souffle

Bush : Est de poursuivre ceux qui la menacent

Ben Laden : Pour faire disparaître le mal

Bush : Le nom de l'opération militaire d'aujourd'hui

Ben Laden : Je jure devant Dieu

Bush : Est Liberté Immuable [...] La paix et la liberté l'emporteront

Ben Laden : Que Dieu nous protège

Bush: Que Dieu nous bénisse ${ }^{91}$ "

La présence dominatrice de ces « dieux » laisse deviner l'impuissance des personnages, engagés malgré eux dans l'événement.

Le chœur, les vers et l'intervention divine semblent éloigner les pièces grecques de la vie quotidienne. Mais, le spectateur contemporain d'une tragédie grecque, et le dramaturge qui se livre à l'adaptation d'un mythe ou d'une tragédie de la Grèce ancienne, puisent les thèmes et l'atmosphère générale des pièces dans un contexte politique moderne. Nous remarquons que l'adaptation du mythe grec d'Antigone au théâtre moderne sous la plume d'Anouilh ou La Guerre de Troie n'aura pas lieu de Giraudoux sont plus conformes à la conception du théâtre politique de Vinaver que les ouvrages antiques originaux.

D'ailleurs, il résume sa conception du théâtre antique en ces termes: "les auvres du passé n'existent que dans le regard que nous portons sur elles. En les regardant nous nous les approprions et les altérons. Nous les utilisons pour notre besoin, au même titre que nous utilisons, de première source, nos perceptions, les images et les réflexions qui nous viennent. ${ }^{92}$ "Sa préoccupation majeure reste le théâtre moderne français.

91 Ibid, pp. 61-71.

92 ID, (1998), op.cit., p.38. 
Ainsi, sans se soumettre à la passivité traditionnelle du spectateur du théâtre bourgeois, le dramaturge crée une surprenante interférence dynamique de la vie quotidienne (le politique) et des questions politiques de plus large envergure (la politique) : c'est ce qu'il appelle "le double visage de la victime pathétique et de l'enfant terrible qui distribue croche-pieds et coups de pied à tout venant. ${ }^{93}$ "

\section{Conclusion:}

Véritable «kaléidoscope théâtral ${ }^{94}$ » qui offre une nouvelle manière de voir aussi bien dramatique que politique, le théâtre de Michel Vinaver se veut un miroir plus grossissant que le cinéma, art en vogue de l'époque.

Sans être expressément subversif, son théâtre reflète l'impact de l'argent, de l'amour et de la guerre qui affectent la vie des gens. Il peut apparaître de prime abord violent, mais par la fragmentation du temps, de l'espace et de l'action, ses pièces invitent à penser aux motifs qui dominent la scène politique. C'est une réflexion sur la réalité politique plutôt qu'un reflet de celle-ci. Vinaver établit des parallèles entre les attitudes individuelles et institutionnelles, montrant comment les relations reflètent le système du pouvoir. Ce qui frappe dans les deux pièces, soumises à notre étude, c'est le sens affirmé d'un quotidien régi par l'histoire. C'est ainsi que son théâtre est vraiment historique, donc politique, lorsqu'il montre non seulement des pièces adaptées de l'antiquité mais aussi des individus contemporains saisis par l'histoire, bien plus que celle faite par les personnages qui seraient censés la faire. L'écriture du quotidien qui incite au montage du contexte historique immédiat n'offre pas de solutions ni de distance à critiquer ; mais permet l'interaction des questions et des réflexions sur les problèmes et les possibilités. Elle rassemble des fragments

93 Ibid, p.32.

${ }^{94}$ ELSTOB K., (1992), op.cit., p.43. 
de temps et d'espace, qui interagissent les uns avec les autres pour peindre une image de plus en plus excitant, diversifiée et troublante d'un pays déchiré par la division, la duplicité et la mauvaise direction.

Ainsi, les paramètres réalistes du théâtre de Vinaver sont distincts, mais son réalisme n'est ni banal ni fiable; il vibre dans un théâtre qui révèle sa propre fragilité, son propre fonctionnement.

Un théâtre de concision, très peu discursif mais qui pointe les tares socio politiques.

Théâtre lucide qui traduit par l'ironie et le sens de l'humour la composition conflictuelle de la patrie ; il révèle la fragilité d'une telle notion.

En établissant, à la manière de Grotowski, le lien étroit entre le spectateur et le dramaturge ou le comédien, les dramaturges ont révolutionné le théâtre de la seconde moitié du vingtième siècle et jusqu'à nos jours. Ils incitent l'auditoire à s'engager à sonder le mystère de la situation présentée par l'intrigue. Mais cette révolution est liée au sens nouveau qu'il convient de donner à la notion même de public et de communication théâtrale. Vinaver fait usage de cette forme d' «exutoires » tolérés par le pouvoir pour représenter généreusement la vie sans se confondre ave elle. L'existence de cette relation fondamentale, liant le théâtre soit à l'histoire, soit à la conjoncture d'une part, et rattachant le spectateur au dramaturge de l'autre sont les deux fondements servant de base à la production théâtrale moderne. 


\section{Bibliographique :}

\section{Corpus et ouvrages de Michel Vinaver:}

Les Coréens, in Théâtre Complet I, (1986). Actes Sud et L'Aire.

11 Septembre 2001, (2002). Paris, L'Arche.

Les Travaux et les jours, (1979). Paris, l'Arche.

Ecrits sur le théâtre I, (1998). Paris, L'Arche.

\section{Articles de Michel Vinaver parus dans des revues:}

(1955). «Les Mythes de la Grèce ancienne, une marche d'approche », in Critique, mars.

(1989). « Ici et maintenant », in L'Art du théâtre, octobre, pp.39- 42

\section{Ouvrages critiques:}

BRADBY D., (1984). Modern French Drama (1940-1980), Cambridge and London, Cambridge University Press.

DAMERVAL G., (1984). Ubu Roi : La bombe comique de 1896, Paris, A.G.

Nizet.

DORT B., (1986). Théâtres, Paris, Seuil.

ELSTOB K.., (1992). The Plays of Michel Vinaver. Political Theatre in

France, NYC, American University studies, Ed. Peter Lang.

GROTOWSKI J. \& FLASZEN L., (1967). L'Institut de recherche sur le jeu de

l'acteur, Wroclaw, éd. du Théâtre Laboratoire. 
GUREWITCH M., (1975). Comedy : the Irrational Vision, NYC, Cornel U.P. JEAN G., (1977). Le Théâtre, Paris, Seuil.

MARKOVIC M., (1974). The Contemporary Marx: Essays on Humanist

Communism, Bristol, New Spokesman Press.

MORTIER D., (1986). Celui qui dit oui, celui qui dit non ou la réception de

Brecht en France (1945-1956), Paris-Genève, Champion-Slatkine.

RABEY D., (1986). British and Irish Political Drama in the Twentieth

Century, London, MacMillan.

SANDIER G., (1982). compte-rendu de Dissident, il va sans dire et Nina, c'est autre chose, Théâtre en crise, Grenoble, La Pensée Sauvage.

TOCQUEVILLE A., « De la démocratie en Amérique 2 », 1840, in Les

Classiques de sciences sociales, éd. PhiloSophie, Chapitre 2, mai 2008.

UBERSFELD A., (1989). Vinaver dramaturge, Paris, Librairie théâtrale.

\section{Chapitres d'ouvrages:}

BARTHES R., (1986). «Préface de Roland Barthes », in Théâtre Complet I de Michel Vinaver, Arles, Actes Sud et L'Aire, pp.37- 41.

BRECHT B., (2000). « Théâtre récréatif ou théâtre didactique ? », in Ecrits sur le théâtre, Paris, Galllimard, Bibliothèque de la Pléiade, pp. 214-216.

\section{V.Articles de revues:}

BARTHES R., (1956) «Aujourd'hui ou les Coréens » (de M. Vinaver, mise en scène par R. Planchon, au théâtre de la Comédie), in France-Observateur, $1^{\text {er }}$ nov.

COPFERMANN E., (1958) «Iphygénie Hôtel», in France-Observateur, 24 avril.

DARGE F., (2009) « Michel Vinaver, dramaturge du réel », propos recueillis par Fabienne Darge, in Le Monde 2, ${ }^{\circ}$ 258, 24-30 janvier.

KIRBY M., (1975) "On Political Theatre", in The Drama Review, $\mathrm{n}^{\circ}$ 19, June. LAMONT R., (1988) «Des petits ébranlements capillaires. L'Art de Michel Vinaver », in Modern Drama, 31 septembre, pp.380-394.

Revue Europe, (avril 2006, n 924, Numéro spécial Michel Vinaver) :

- RYNGAERT J-P., (2006) «L'Ironie contre l'esprit de sérieux. Présence du comique chez Michel

Vinaver »

- THOMASSEAU J-M., (2006) «L'ouï-dire et

l'inouï »

UBERSFELD A., (2006) «Vinaver et le cœur»

\section{Webographie:}

- DIAZ S., "'Réfléchir l'événement plutôt qu'y réfléchir' : l'imitation du 11 septembre 2001 dans la pièce de Michel Vinaver», Agôn [En ligne], Dossiers, (2011) HS n ${ }^{\circ} 1$ : Mettre en scène l'événement, 11 Septembre 2001 de Michel 
Vinaver, mis à jour le : 31/10/2011.

URL : http://agon.ens-lyon.fr/index.php?id=1803. consulté 18/11/2017.

- DIAZ S. \& NOEL A-S., «Les Troyennes, tragédie new-yorkaise ?», Agôn[En ligne], Dossiers, (2011) HS n ${ }^{\circ} 1$ : Mettre en scène l'événement, 11 septembre 2001 de Michel Vinaver, mis à jour le : 23/12/2011,

URL : http://agon.ens-lyon.fr/index.php?id=1782. consulté le 17/9/2017.

- Babelio https://www.babelio.com/livres/Vinaver-11-septembre-2001/175909, consulté 28/10/2017.

- Académie de Paris, Pièce démontée, Théâtre de la ville, $\mathrm{n}^{\circ} 1$ « Les dossiers pédagogiques « Théâtre » et «Arts du cirque » du réseau SCEREN en partenariat avec le Théâtre de la Ville, pp.1-29.

http://crdp.ac-paris.fr/piece-demontee/pdf/11-sept_total.pdf consulté 2/3/2018 ,p.1

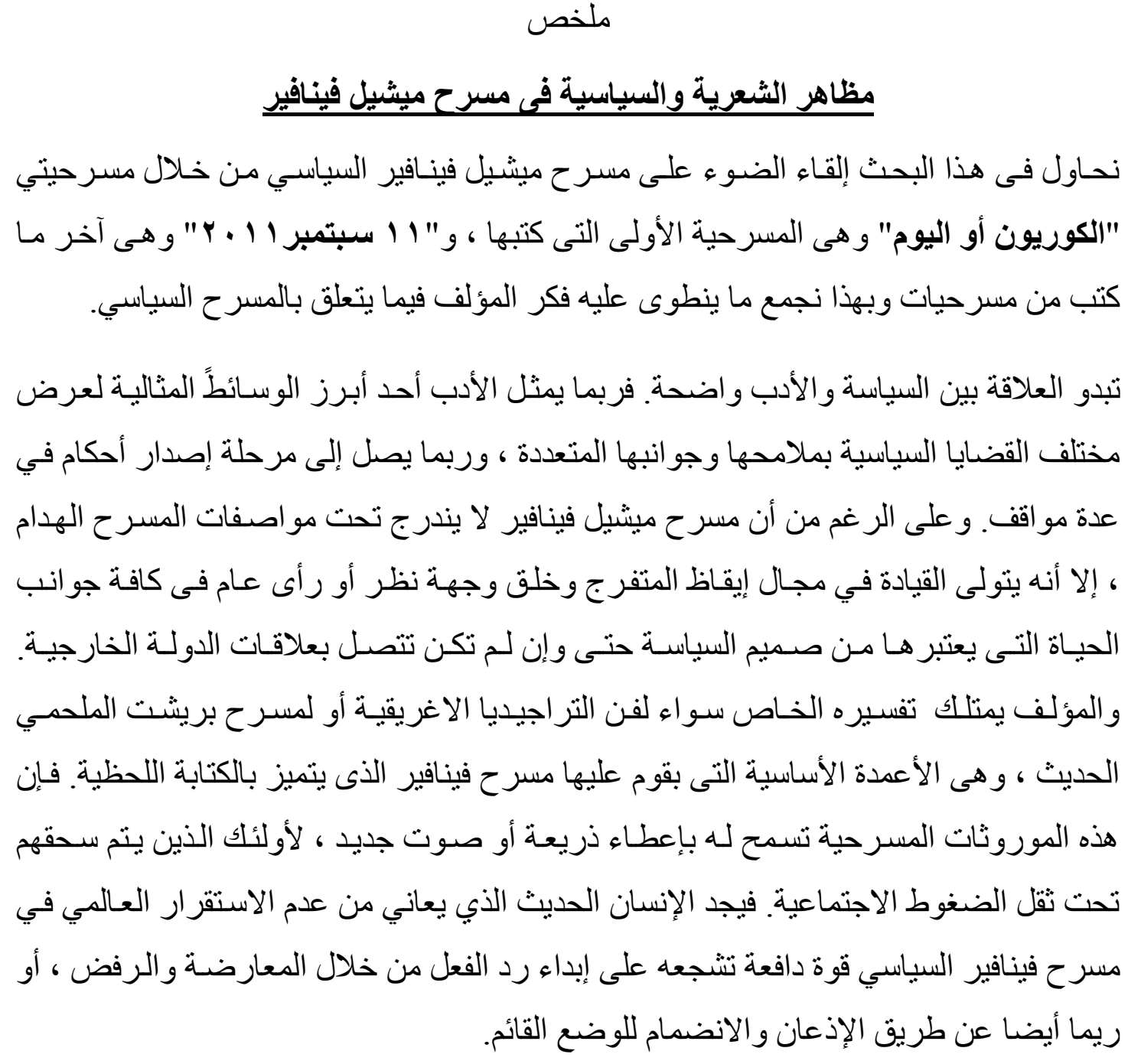




\section{RESUME}

\section{Poétique et politique dans le théâtre de Michel Vinaver}

La relation entre la politique et la littérature est évidente; ce dernier peut offrir un médium parfait qui représente les enjeux, les avatars des problèmes politiques, et peut éventuellement porter des jugements sur plusieurs situations. Bien que n'étant pas subversif, le théâtre de Michel Vinaver occupe une place de choix dans ce domaine. Son interprétation particulière, soit de l'ancienne tragédie, soit du théâtre moderne brechtien épique, lui permet de donner un alibi, une nouvelle voix à ceux qui sont écrasés sous le poids des pesanteurs socio culturelles. L'homme moderne affligé par l'instabilité mondiale trouve dans le théâtre politique de Vinaver une force motrice qui encourage ses réactions soit par l'opposition, le refus, ou par l'acquiescement et l'adhésion. 